\title{
Experimental characterization of autonomous heat engine based on minimal dynamical-system model
}

\author{
Shoichi Toyabe (1) ${ }^{1}$ and Yuki Izumida ${ }^{2, *}$ \\ ${ }^{1}$ Department of Applied Physics, Graduate School of Engineering, Tohoku University, Sendai 980-8579, Japan \\ ${ }^{2}$ Department of Complexity Science and Engineering, Graduate School of Frontier Sciences, The University of Tokyo, \\ Kashiwa 277-8561, Japan
}

(Received 7 November 2019; accepted 10 July 2020; published 27 July 2020; corrected 19 August 2020)

\begin{abstract}
The autonomous heat engine is a model system of autonomous nonequilibrium systems, exploiting nonequilibrium flow for operations. As the Carnot engine has essentially contributed to the equilibrium thermodynamics, an autonomous heat engine is expected to play a critical role in the challenge of constructing nonequilibrium thermodynamics. However, the high complexity of the engine, involving an intricate coupling among heat, gas flow, and mechanics, has prevented simple modeling. Here, we experimentally characterized the nonequilibrium dynamics and thermodynamics of a low-temperature-differential Stirling engine, which is a model autonomous heat engine. Our experiments demonstrated that the core engine dynamics are quantitatively described by a minimal dynamical model with only two degrees of freedom. The model proposes a novel concept that illustrates the engine as a thermodynamic pendulum driven by a thermodynamic force. This work describes an approach to exploring the nonequilibrium thermodynamics of autonomous systems based on a simple dynamical system.
\end{abstract}

DOI: 10.1103/PhysRevResearch.2.033146

\section{INTRODUCTION}

The characterization of autonomous nonequilibrium systems is one of the challenging tasks of modern physics. These systems are typically complex, but there has been a long pursuit toward deriving universal and simple relations governing them. For this purpose, the extension of thermodynamics would be a promising approach because thermodynamics illustrates a universal structure of the system behind the details. Thermodynamics is originally formulated based on infinitely slow quasistatic processes [1]. The recent challenge in constructing the finite-time thermodynamics tries to characterize the thermodynamic quantities of nonequilibrium systems by incorporating the finite-speed dynamics. The finite-time thermodynamics was already successful in characterizing the efficiency at maximum power [2-9], optimal control with minimal energy cost [10-12], the tradeoff relations between power and efficiency [13-17], and stochastic heat engines [18-20].

A simple model is always the basis of scientific understanding. Carnot's heat engine is one of the most prominent examples in history and has played an essential role in constructing thermodynamics [21]. As a natural extension, along with this approach, autonomous heat engines are expected to play essential roles in the development of finite-time thermodynamics. The Carnot engine requires control by an external agent, whereas autonomous heat engines implement

\footnotetext{
*izumida@k.u-tokyo.ac.jp

Published by the American Physical Society under the terms of the Creative Commons Attribution 4.0 International license. Further distribution of this work must maintain attribution to the author(s) and the published article's title, journal citation, and DOI.
}

autonomous regulation. However, the high complexity of the engine dynamics involving a mechanical motion, heat flow, and gas flow has prevented simple modeling.

Here, we experimentally characterize the nonequilibrium dynamics of a low-temperature-differential Stirling engine (LTD-SE), which is a model autocatalytic heat engine with a minimal structure (Fig. 1) [22]. We primarily focus on the bifurcation dynamics because the bifurcation behavior characterizes the system's universal properties behind the details. We deduce a simple two-variable model of this engine from the experimental results based on the previously proposed theoretical framework [23]. Such simple modeling would enable us to build a theoretical framework of autonomous heat engines towards the establishment of the finite-time thermodynamics.

The Stirling engine is an autonomous and closed heat engine [24-27]. Given a temperature difference, the engine cycles the volume, temperature, and pressure inside a cylinder autonomously without external timing control and rotates a flywheel unidirectionally. Theoretically, an ideal Stirling engine, i.e., a Stirling cycle, achieves the Carnot efficiency. The LTD-SE [22,28] consists of a power piston, displacer, flywheel, two cranks, and two rods connecting the piston and displacer to the flywheel [Fig. 1(a)]. The flywheel rotates when a sufficiently large temperature difference is given between the top and bottom plates of the cylinder. The flywheel rotation is synchronized with the oscillation of the internal displacer and the power piston. The displacer serves to switch the heat baths between the top and bottom plates. Thus, the gas temperature and pressure oscillate and move the power piston up and down. This piston motion drives the flywheel rotation. The $\pi / 2$ phase difference of the displacer and the power piston makes a cycle. The flywheel provides inertia necessary for a smooth rotation. When the opposite temperature difference is 

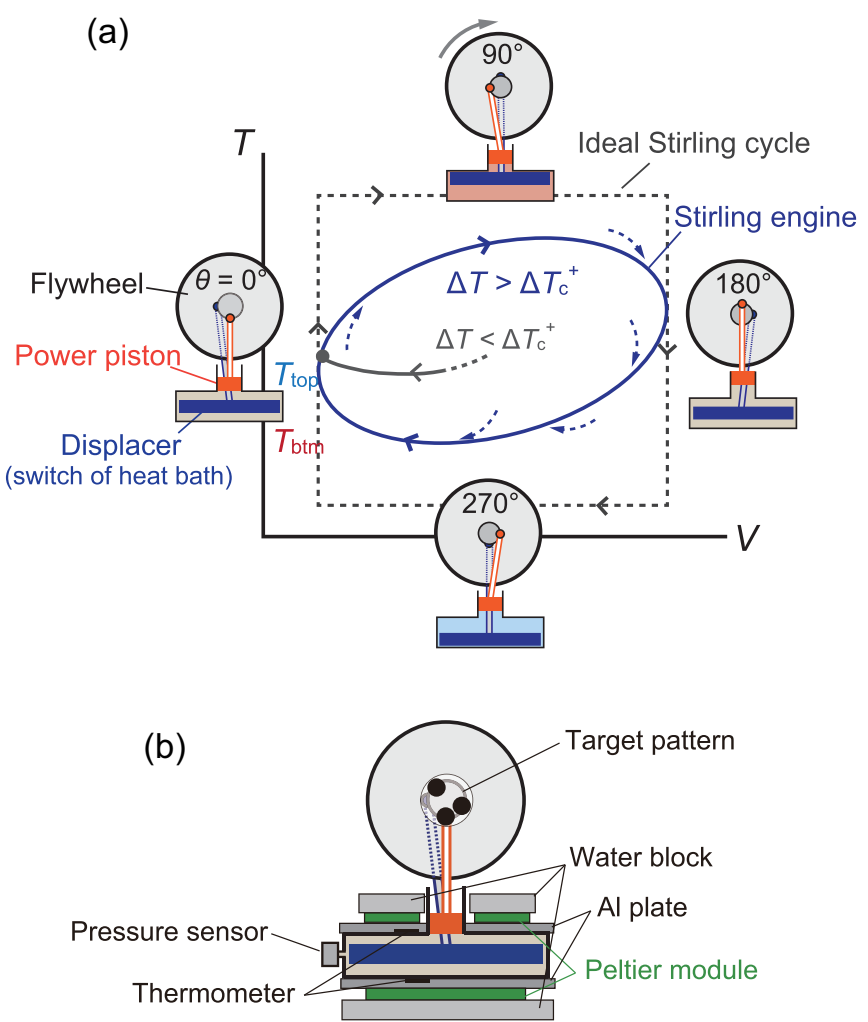

FIG. 1. Schematic of the experiments. (a) Thermodynamic diagram of the low-temperature-differential Stirling engine. The heating from the bottom increases the internal pressure, pushes the power piston upward, and drives the flywheel rotation, which then pushes the displacer downward. The displacer serves to switch the heat baths. When the displacer moves downward, most gas in the cylinder moves to the upper side and makes contact with the top plate at a lower temperature of $T_{\text {top }}$. The cooling of gas results in the pressure decrease and pushes the power piston downward, and the cycle restores to the initial state. The rotation can be inverted by an opposite temperature difference. (b) Experimental setup. The temperatures at the top and bottom plates are controlled by Peltier modules. The pressure difference between the outside and inside of the cylinder is monitored by a differential pressure sensor. The rotation of the flywheel is monitored at $100 \mathrm{~Hz}$ by videoscopy of the target pattern (three circles aligned in an isosceles triangle configuration) attached to the crank screw.

given, the flywheel rotates in the opposite direction with an inverted mechanism.

\section{EXPERIMENT}

An LTD-SE (N-92 type) was bought from Kontax (UK). We controlled the temperatures at the top and bottom plates, $T_{\text {top }}$ and $T_{\mathrm{btm}}$, of the cylinder [Fig. 1(b)] and monitored the angular position $\theta(t)$ and angular velocity $\omega(t)$ of the flywheel and the pressure $p(t)$ inside the chamber. See the Materials and Methods section for details.

\section{A. Rotation}

Without stimulation, the engine was settled at a stationary position $\theta \simeq-38^{\circ}$, where the pressure difference across the (a)
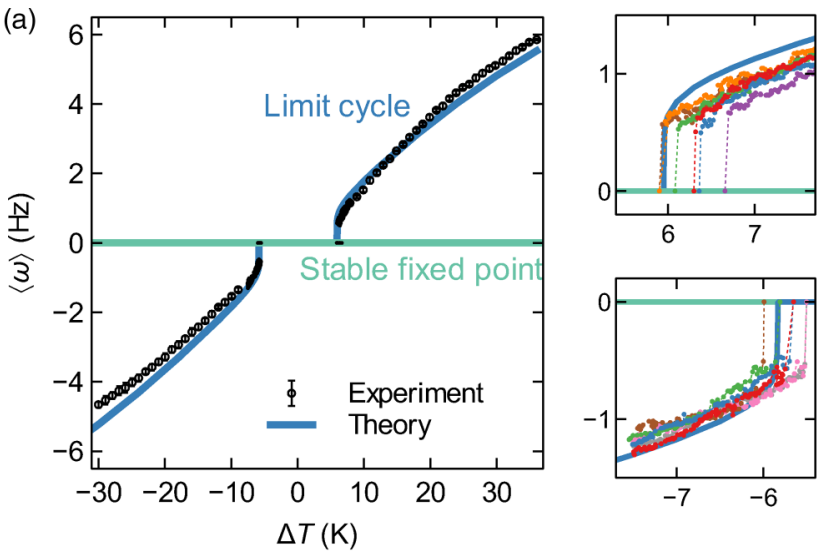

(b)
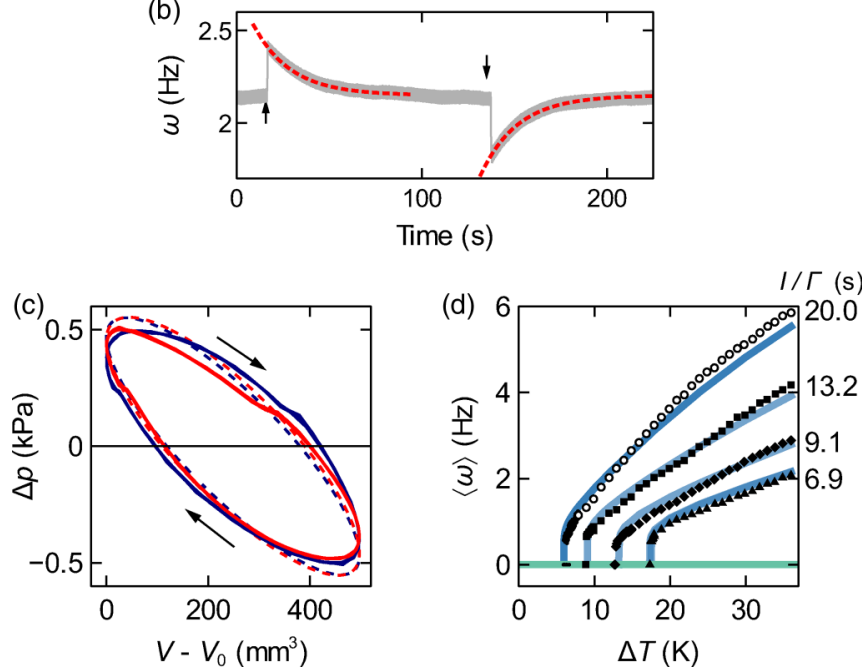

FIG. 2. Steady rotation. (a) The time-averaged angular velocity $\langle\omega\rangle$ of the limit cycle was plotted against the temperature differences $\Delta T$. At $|\Delta T| \geqslant 8 \mathrm{~K}$, three experimental traces were averaged (circle). The error bar corresponds to one standard deviation. At $|\Delta T|<$ $8 \mathrm{~K}$, twelve traces were superposed (solid lines, six for $\Delta T>0$ and six for $\Delta T<0$ ). (b) The stability of the rotation state at $\Delta T=$ $12 \mathrm{~K}$. The rotation state is stable against perturbations (indicated by arrows). Dashed lines are fitting curves by exponential functions, of which the time constant corresponds to $I / \Gamma$. (c) Pressure-volume curves for $\Delta T=12 \mathrm{~K}$ (red) and $-12 \mathrm{~K}$ (blue) obtained by experiments (solid) and theories (dashed). The cycling direction was clockwise independent of the sign of $\Delta T$. The average of $\Delta p$ for the theoretical curves was forced to zero. (d) The time-averaged angular velocity $\langle\omega\rangle$ without (open) or with (closed) additional frictional load. The solid curves are numerical simulations with $\Gamma$ obtained by measuring the time constant $I / \Gamma$ to the perturbation (panel (b) and Fig. S2 [29]).

power piston and the gravity force on the power piston, displacer, crank screws, and rods are presumably balanced. When an initial angular momentum with a sufficiently large magnitude was given, the flywheel rotated steadily with an angular velocity determined by $\Delta T=T_{\mathrm{btm}}-T_{\text {top }}$ when $T_{\text {top }}$ is fixed [Fig. 2(a)]. The rotation direction changed depending on the sign of $\Delta T$. When the engine in this steady state was perturbed by hand, the angular velocity was soon recovered to the steady rate [Fig. 2(b)], implying a stable limit cycle. 
The pressure-volume curve exhibited a circular shape [Fig. 2(c)], demonstrating a heat engine. The cycling direction in the PV diagram was the same independent of the sign of $\Delta T$, and the PV curves were nearly symmetric for the sign of $\Delta T$. The area increased with $|\Delta T|$ (Fig. S1 of the Supplemental Material [29]).

The time-averaged steady angular velocity $\langle\omega\rangle$ changed nearly linearly with $\Delta T$ [Fig. 2(a)]. $|\langle\omega\rangle|$ decreased with $|\Delta T|$ and vanished at a finite value of $\Delta T$. The threshold value, $\Delta T_{\mathrm{c}}$, was slightly different for the sign of $\Delta T ; \Delta T_{\mathrm{c}}^{+}=$ $6.2 \pm 0.3 \mathrm{~K}$ and $\Delta T_{\mathrm{c}}^{-}=-5.7 \pm 0.2 \mathrm{~K}$ (mean \pm standard deviation), indicating the asymmetry of the dynamics for the sign of $\Delta T$.

The stalling at $\Delta T_{\mathrm{c}}^{ \pm}$was accompanied by a steep change in $\langle\omega\rangle$, implying a homoclinic bifurcation [30]. The homoclinic bifurcation is a kind of a global bifurcation, and is seen in, for example, a driven pendulum and a Josephson junction. A stable limit cycle disappears with a steep but continuous transition at the homoclinic bifurcation point. However, such the continuity is too steep to be observed in the experiments because $\omega$ is inversely proportional to $-\ln \left|\Delta T-\Delta T_{c}^{ \pm}\right|$for $|\Delta T|>\left|\Delta T_{\mathrm{c}}^{ \pm}\right|$in the vicinity of the bifurcation point [30]. Instead, discontinuous change in $\langle\omega\rangle$ was observed.

We also induced additional frictional load by pressing a brush for Chinese calligraphy to the flywheel. The increase in load suppressed the angular velocity and increased $\Delta T_{\mathrm{c}}^{+}$ [Fig. 2(c)].

\section{B. Bifurcation analysis}

We characterize the bifurcation dynamics in detail. Figure 3(a) shows two typical trajectories started with different initial angular velocities at $\Delta T>\Delta T_{\mathrm{c}}^{+}$. With a large initial angular velocity, we observed the convergence to the periodic trajectory determined by $\Delta T$. As noted, the periodic trajectory was stable against perturbation and was identified as a stable limit cycle [Fig. 2(b)].

With a small initial angular velocity, the trajectory was first attracted to $\mathrm{U}$ at $(\theta, \omega) \simeq\left(153^{\circ}, 0\right)$ and then collapsed to $S$ at $(\theta, \omega) \simeq\left(-38^{\circ}, 0\right)$ in a spiral-shaped manner, failing in converging to the stable limit cycle. When $\Delta T_{\mathrm{c}}^{-}<\Delta T<$ $\Delta T_{\mathrm{c}}^{+}, \mathrm{S}$ was the unique stable attractor [Fig. 3(b)]. These results suggest that $\mathrm{U}$ and $\mathrm{S}$ are a saddle point and a stable fixed point (spiral), respectively, and that $\mathrm{S}$ and the stable limit cycle coexist for $\Delta T>\Delta T_{\mathrm{c}}^{+}$and $\Delta T<\Delta T_{\mathrm{c}}^{-}$.

Figure 3(c) shows the trajectories of the steady rotations, $\omega(\theta)$, at various $\Delta T$ above the threshold. $\omega(\theta)$ was relatively flat at large $|\Delta T|$ and exhibited a rugged profile at small $|\Delta T|$. Specifically, as $\Delta T$ approaches $\Delta T_{c}$, a part of the limit cycle approaches the saddle point $\mathrm{U}$, which is one of the characteristics of the homoclinic bifurcation.

All the characteristics observed above indicate the homoclinic bifurcation of the limit cycle at $\Delta T_{\mathrm{c}}$ [30] and controvert other possibilities, including the Hopf bifurcation where local stability of the fixed point alters at the bifurcation point. We will analyze the experimental data based on simple dynamical-system modeling below in the Theoretical Analysis section.
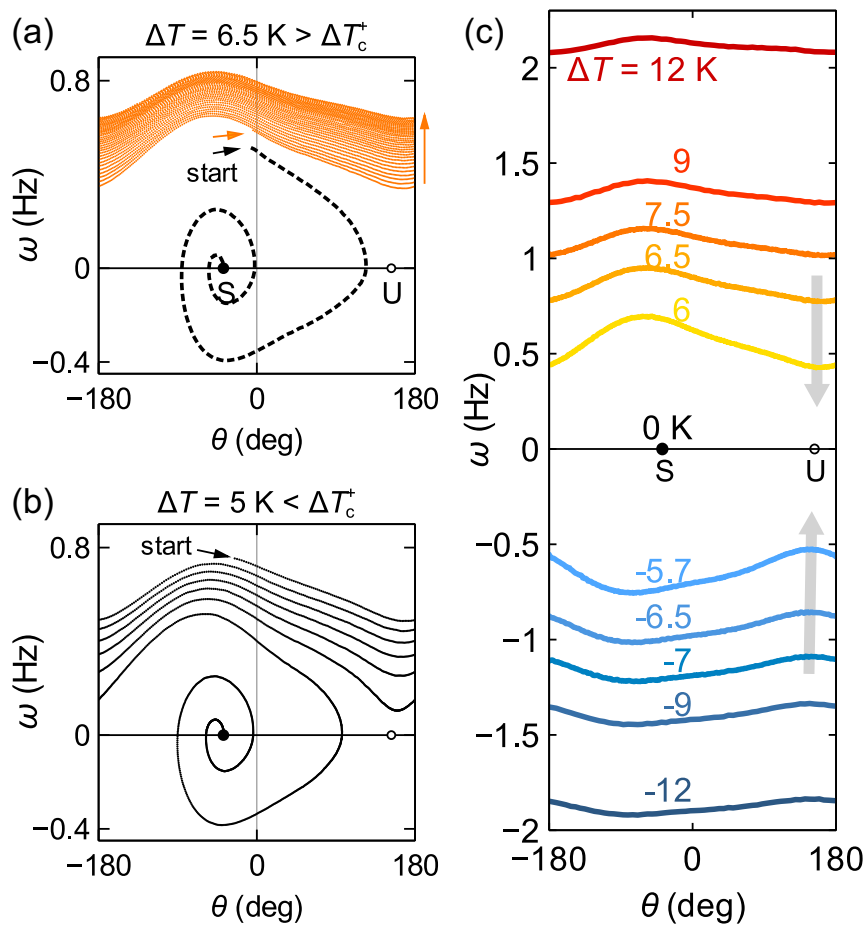

FIG. 3. Rotational trajectories. (a) Rotational trajectories at $\Delta T=6.5 \mathrm{~K}>\Delta T_{\mathrm{c}}^{+}$initiated with small (dashed, black) or large (solid, orange) angular velocity $\omega$. With a large initial angular velocity, $\omega$ gradually increased and the engine settled down to a steady rotation. With a small initial angular velocity, the engine stopped the rotation at the stable fixed point $\mathrm{S}$ at $(\theta, \omega) \simeq\left(-38^{\circ}, 0\right)$ (closed circle) after passing nearby the saddle point $U$ at $(\theta, \omega) \simeq\left(153^{\circ}, 0\right)$ (open circle). See Fig. 1(a) for the definition of $\theta$. (b) Rotational trajectory at $\Delta T=5 \mathrm{~K}<\Delta T_{\mathrm{c}}^{+}$. (c) Steady rotational trajectories at different $\Delta T$.

\section{Oscillatory mode}

We also discovered an oscillation branch at $\Delta T \leqslant-27 \mathrm{~K}$ [Fig. 4(a)]. Here, for exploring a small $\Delta T$ region, $T_{\text {top }}$ was set to a relatively large value, $65{ }^{\circ} \mathrm{C}$. When we shifted the flywheel angle slightly from $\mathrm{S}$ gently by hand, the flywheel started a periodic oscillation with a finite amplitude and a period of about 10 seconds, which can be considered an oscillatory stable limit cycle.

This limit cycle showed complicated behaviors; the amplitude increases accompanied by a period-doubling bifurcation $(-27.5 \mathrm{~K} \geqslant \Delta T>-33.5 \mathrm{~K})$, seemingly aperiodic oscillation similar to chaos $(-33.5 \mathrm{~K} \geqslant \Delta T>-39.5 \mathrm{~K})$, and again periodic oscillations accompanied by small additional oscillations $(-39.5 \mathrm{~K} \geqslant \Delta T>-61.5 \mathrm{~K})$. The oscillation branch disappeared at $\Delta T \leqslant-61.5 \mathrm{~K}$, and a small perturbation got drawn into a rotation branch. The rotation mode was observed for all $\Delta T<0$ with a sufficiently large initial angular velocity. The oscillation was not observed for $\Delta T>$ 0 . The stable fixed point (spiral) at $\mathrm{S}$ became unstable at $\Delta T=-39.5 \mathrm{~K}$ (Fig. S3 [29]), indicating that a subcritical Hopf bifurcation accompanied by the disappearance of an unstable limit cycle occurred. Although the unstable limit cycle was difficult to identify by experiments, we may expect that the oscillatory stable limit cycle and the unstable limit 

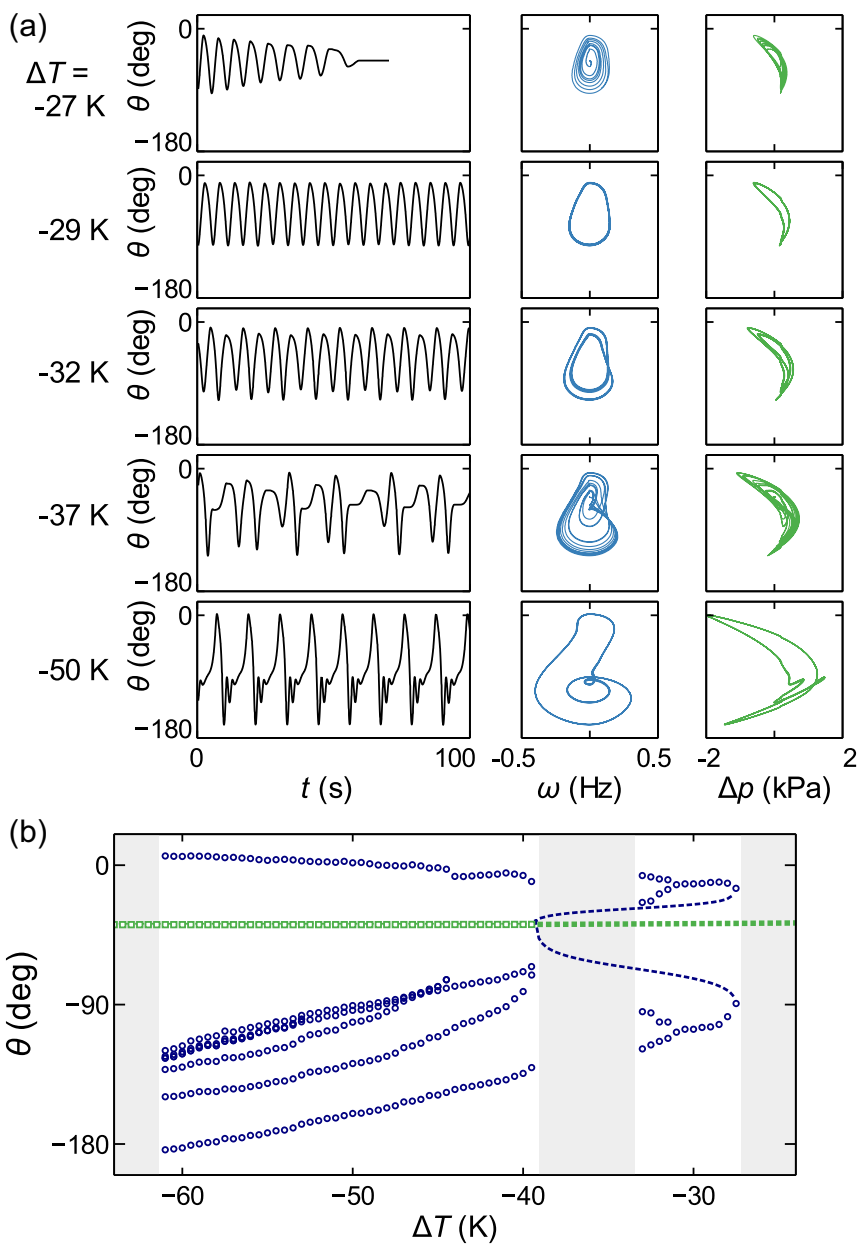

FIG. 4. Oscillation mode observed at $T_{\text {top }}=65^{\circ} \mathrm{C}$. (a) $\theta$ as functions of time (left), $\omega$ (center), and $\Delta p$ (right). (b) Peak angles of the oscillation (circle), stable fixed points (closed square), unstable fixed points (open square), and expected unstable limit cycles (dashed line).

cycle were created in a pair at $\Delta T=-27.5 \mathrm{~K}$ [30]. We need further studies to determine the bifurcation characteristics of the oscillation branch, including the onset and disappearance of the seemingly aperiodic oscillation.

\section{THEORETICAL ANALYSIS}

For deducing the model that explains the experimental observations, we compared the above results with the theory proposed recently [23]. The theory describes the flywheel rotation with a simple equation of motion with only two variables:

$$
\begin{aligned}
\dot{\theta} & =\omega, \\
I \dot{\omega} & =s\left[p(\theta, \omega)-p_{0}\right] r \sin \theta-\Gamma \omega .
\end{aligned}
$$

Here, $I$ and $\Gamma$ are the moment of inertia and frictional coefficient, respectively, of the engine's rotational degree of freedom. $r$ is the crank radius. $s$ is the sectional area of the power piston. $s\left[p(\theta, \omega)-p_{0}\right] \equiv s \Delta p$ corresponds to the force on the crank applied by the power piston via a rod, and $s \Delta p \cdot r \sin \theta$ is the torque on the flywheel (a piston-crank mechanism). $p_{0}$ is external pressure.

The theory [23] approximates that the gas is in contact with a single heat bath at an effective temperature $T_{0}+\sin \theta \frac{\Delta T}{2}$, where $T_{0}=\left(T_{\text {top }}+T_{\mathrm{btm}}\right) / 2$. The effective temperature oscillates between $T_{\text {top }}$ and $T_{\text {btm }}$ synchronized with the displacer motion. For the quantitative analysis of the experimental data based on (1), we modeled the system simply as

$$
\begin{gathered}
T(\theta, \omega)=T_{0}+\alpha \sin (\theta-\omega \tau) \frac{\Delta T}{2}, \\
p(\theta, \omega)=\beta \frac{n R T(\theta, \omega)}{V(\theta)} .
\end{gathered}
$$

Here, the effect of the heat transfer on the gas temperature $T(\theta, \omega)$ is simply implemented by two parameters: the magnitude $\alpha$ and the time delay $\tau$ under an adiabatic assumption that the temperature equilibration is sufficiently fast compared to the flywheel dynamics. $p(\theta, \omega)$ is calculated based on an effective equation of the state for the ideal gas. $n$ is the amount of substance of the internal gas, and $R$ is the gas constant. $V(\theta)=V_{0}+r s(1-\cos \theta)$ is the volume of the cylinder, where $V_{0}$ is the cylinder volume excluding the displacer volume. The temperature and pressure may be nonuniform inside the cylinder, and therefore the equation of the state for the ideal gas may not hold as it is. The coefficient $\beta$ is introduced to compensate for such an effect.

The two-variable model (1) with (2) and (3) reproduced the $\Delta T$ dependence of $\langle\omega\rangle$ well quantitatively [Fig. 2(a)] including the steep change in the vicinity of $\Delta T_{\mathrm{c}}$ and the pressure-volume curve. See Materials and Methods for the parameters used. The model also succeeded in reproducing the bifurcation curves for the increased frictional load [Fig. 2(d)]. Here, we used the same parameters except for the frictional coefficients, which were evaluated from the response curves under each condition (Fig. S2 [29]). Note that the model (1)(3) exhibits the homoclinic bifurcation as $|\Delta T|$ is decreased, where a stable limit cycle disappears at $\Delta T_{\mathrm{c}}^{ \pm}$by colliding with a saddle point [23]. This is consistent with the experimental suggestions [arrows in Fig. 3(c)]. These results validate the model (1). This two-variable model is a minimal model of autonomous heat engines in the sense that at least two variables are required to describe a limit cycle.

On the other hand, the oscillation branch (Fig. 4) was not observed by this minimal model. At $\Delta T \leqslant-31.5 \mathrm{~K}$, the trajectory $\theta(\omega)$ possessed an intersection [Fig. 4(a)], meaning that the description by only $\theta$ and $\omega$ no longer describes the oscillation dynamics correctly at some points. Specifically, $p(\theta, \omega)$ was a multiple-valued function of $(\theta, \omega)$ at the intersection points, suggesting that (3) is not valid at these points.

\section{DISCUSSION}

An autonomous heat engine is a model system of autonomous nonequilibrium systems. The model (1) reproduces the essential characteristics of the engine, including the bifurcation dynamics and the thermodynamic diagram despite its simplicity. The model contains $\Delta T$ explicitly through $p(\theta, \omega)$ [23], proposing the concept that the LTD-SE is a thermodynamic pendulum driven by a thermodynamic force characterized by $\Delta T$. Whereas the model is derived based 
on the LTD-SE, this simple and intuitive formulation is expected to be applicable to a wide range of autonomous heat engines with small modifications of, for example, the cycle shape $T(\theta, \omega)$ and the piston-crank mechanism $r \sin \theta$. The model does not reproduce the oscillation branch. Although the oscillation is not an essential operation mode of the engine, it would be intriguing to explore what modification to the theory could successfully describe the oscillation.

The formulation of the thermodynamic efficiency of the autonomous heat engine would be of crucial importance, which is complementary to the formulation in nonautonomous heat engines $[2,3]$. The evaluation of efficiency requires the measurement of the heat flowing through the engine and remains for future studies.

\section{CONCLUSION}

We demonstrated that the essential characteristics of the complex autonomous heat engine are reproduced by a minimal and intuitive two-variable model (1) quantitatively. The present work supports an approach to exploring the finitetime thermodynamics of autonomous heat engines based on a simple dynamical-system description.

The Stirling engine is attracting growing attention in industries because it can utilize low-grade heating sources such as solar power, waste heat in the industries, and geothermal energy, and also is environmentally friendly. Because of its autonomous, clean, and simple machinery, the use of the Stirling engine for generating electric power for spacecraft is being considered [25]. Nevertheless, the physics behind engine dynamics has not been explored extensively. Our experiments succeeded in characterizing the bifurcation mechanism. Such an approach based on physics would be effective in improving engine performance.

\section{MATERIALS AND METHODS}

\section{A. Experimental setup}

An LTD-SE (N-92 type) was bought from Kontax (UK). The temperatures of the top and bottom plates of the cylinder were controlled by Peltier modules equipped with water flowing blocks [Fig. 1(b)]. The temperatures were monitored at $2.5 \mathrm{~Hz}$ by platinum resistance temperature detectors attached to the surfaces of the plates. A target pattern (three circles aligned in an isosceles triangle configuration) was attached to the crank screw connected to the displacer for monitoring the angular position of the flywheel [Fig. 1(b)]. The image of the target pattern was recorded by a high-speed camera (Basler, Germany) at $100 \mathrm{~Hz}$ and analyzed in real time to obtain the angular position and the angular velocity of the flywheel. A pressure sensor (Copal Electronics, Japan) was fixed at the side of the cylinder to monitor the inner pressure. We monitored the angular position $\theta(t)$, angular velocity $\omega(t)$ of the flywheel, and the pressure $p(t)$ inside the chamber under controlled $\Delta T(t)$. All the experiments were controlled by a computer equipped with a program developed on LABVIEW (National Instruments).

\section{B. Bifurcation dynamics}

For evaluating $\Delta T$ dependence of the angular velocity [Fig. 2(a)], we manually provided an initial angular momentum at $\Delta T=36$ or $-30 \mathrm{~K}$, keeping $T_{\text {top }}=24^{\circ} \mathrm{C}$, and waited for about one hour for sufficient relaxation of the temperatures and flywheel rotation. Then, keeping $T_{\text {top }}=24^{\circ} \mathrm{C}$, we varied $\Delta T$ from 36 to $0 \mathrm{~K}$ or from $-30 \mathrm{~K}$ to $0 \mathrm{~K}$ in a stepwise manner at a rate of $\pm 1 \mathrm{~K}$ every $180 \mathrm{~s}$ for $|\Delta T|>8 \mathrm{~K}$ and $\pm 0.02 \mathrm{~K}$ every $60 \mathrm{~s}$ or $120 \mathrm{~s}$ otherwise.

\section{Parameters for theoretical curves}

We used the following parameters for the theoretical curves in Figs. 2(a), 2(c), and 2(d). $V_{0}=44900 \mathrm{~mm}^{3}, s=71 \mathrm{~mm}^{2}$, $r=3.5 \mathrm{~mm}, I=5.7 \times 10^{-5} \mathrm{~kg} \mathrm{~m}^{2}$, and $p_{0}=101.3 \mathrm{kPa} . n=$ $0.00185 \mathrm{~mol}, R=8.314 \mathrm{~J} / \mathrm{Kmol}$. We determined $\alpha, \beta$, and $\tau$ as $0.17,0.94$, and $15 \mathrm{~ms}$, respectively, by fitting. The friction coefficient $\Gamma$ was measured by evaluating the time constant after a perturbation (Fig. 2(b) and S2 [29]). The time constant is approximately given by $I / \Gamma$.

\section{ACKNOWLEDGMENT}

This work was supported by JSPS KAKENHI Grants No. JP18H05427 and No. JP19K03651.
[1] H. Callen, Thermodynamics and an Introduction to Thermostatistics, 2nd ed. (Wiley, New York, NY, 1985).

[2] F. Curzon and B. Ahlborn, Am. J. Phys. 43, 22 (1975).

[3] P. Salamon, J. D. Nulton, G. Siragusa, T. R. Andersen, and A. Limon, Energy 26, 307 (2001).

[4] C. Van den Broeck, Phys. Rev. Lett. 95, 190602 (2005).

[5] B. Jiménez de Cisneros and A. C. Hernández, Phys. Rev. Lett. 98, 130602 (2007).

[6] M. Esposito, K. Lindenberg, and C. Van den Broeck, Phys. Rev. Lett. 102, 130602 (2009).

[7] M. Esposito, R. Kawai, K. Lindenberg, and C. Van den Broeck, Phys. Rev. Lett. 105, 150603 (2010).

[8] G. Benenti, K. Saito, and G. Casati, Phys. Rev. Lett. 106, 230602 (2011).
[9] Y. Izumida and K. Okuda, Phys. Rev. Lett. 112, 180603 (2014).

[10] T. Schmiedl and U. Seifert, Phys. Rev. Lett. 98, 108301 (2007).

[11] I. A. Martínez, A. Petrosyan, D. Guéry-Odelin, E. Trizac, and S. Ciliberto, Nat. Phys. 12, 843 (2016).

[12] S. Tafoya, S. J. Large, S. Liu, C. Bustamante, and D. A. Sivak, Proc. Natl. Acad. Sci. USA 116, 5920 (2019).

[13] K. Brandner, K. Saito, and U. Seifert, Phys. Rev. X 5, 031019 (2015).

[14] N. Shiraishi, K. Saito, and H. Tasaki, Phys. Rev. Lett. 117, 190601 (2016).

[15] O. Raz, Y. Subaşı, and R. Pugatch, Phys. Rev. Lett. 116, 160601 (2016).

[16] M. Polettini and M. Esposito, Europhys. Lett. 118, 40003 (2017). 
[17] P. Pietzonka and U. Seifert, Phys. Rev. Lett. 120, 190602 (2018).

[18] M. Serra-Garcia, A. Foehr, M. Molerón, J. Lydon, C. Chong, and C. Daraio, Phys. Rev. Lett. 117, 010602 (2016).

[19] I. A. Martínez, E. Roldán, L. Dinis, D. Petrov, J. M. R. Parrondo, and R. A. Rica, Nat. Phys. 12, 67 (2016).

[20] V. Blickle and C. Bechinger, Nat. Phys. 8, 143 (2011).

[21] S. Carnot, Reflections on the Motive Power of Heat, edited and translated by R. H. Thurston (Wiley, New York, NY, 1897).

[22] J. R. Senft, An Introduction to Low Temperature Differential Stirling Engines (Moriya Press, River Falls, Wisconsin, 2008).

[23] Y. Izumida, Europhys. Lett. 121, 50004 (2018).

[24] J. R. Senft, An Introduction to Stirling Engines (Moriya Press, River Falls, Wisconsin, 1993).

[25] M. Wolverton, Sci. Am. 298, 22 (2008).
[26] B. Kongtragool and S. Wongwises, Renew. Sustain. Energy Rev. 7, 131 (2003).

[27] J. W. L. Köhler, Sci. Am. 212, 119 (1995).

[28] Y. J. Lu, H. Nakahara, and J. S. Bobowski, Phys. Teach. 58, 18 (2020).

[29] See Supplemental Material at http://link.aps.org/supplemental/ 10.1103/PhysRevResearch.2.033146 for figures including the pressure-volume curves and relaxation curves at different temperature differences and the conversion to the oscillatory stable limit.

[30] S. H. Strogatz, Nonlinear Dynamics and Chaos: With Applications to Physics, Biology, Chemistry, and Engineering, 2nd ed. (Westview, Boulder, 2014).

Correction: The surname of the first author contained an error and has been fixed. 